BIO -GRAFÍA ESCRITOS SOBRE LA BIOLOGÍA Y SU ENSEÑANZA. EDICIÓN EXTRA-ORDINARIA.

MEMORIAS DEL I CONGRESO NACIONAL DE INVESTIGACIÓN EN

ENSEÑANZA DE LA BIOLOGÍA. VI ENCUENTRO NACIONAL DE INVESTIGACIÓN EN ENSEÑANZA DE LA BIOLOGÍA Y LA EDUCACIÓN

AMBIENTAL. ISSN 2027 1034. P. P. 233-242.

\title{
LOS MICROMUNDOS DE LOS ESTUDIANTES DE ESCUELAS RURALES Y URBANAS RELACIONADOS CON AMBIENTE Y NATURALEZA.
}

\section{STUDENTS FROM RURAL AND URBAN SCHOOLS RELATED TO ENVIRONMENT AND NATURE MICROWORLDS.}

\section{POR: ARCELIO VELASCO R.}

\section{Resumen}

La ponencia se basa en una investigación alrededor de los sentidos, significados y espacios relevantes que construyen los estudiantes en cuanto al ambiente y la naturaleza en escuelas de nivel primario (rurales y urbanas), se realiza desde el enfoque de la investigación-acción y la cartografía social. Se muestra la importancia de recoger los significados y sentidos a la hora de realizar propuestas ambientales escolares con miras a lograr un diálogo entre lo que se sabe y lo que se aprende, en contextos particulares.

\section{Abstract}

The paper is based on a research about the senses, meanings and relevant spaces that students build about the environment and nature in (rural and urban) elementary schools. It was carried out from the approach Action research social mapping. It shows the importance of gathering meanings and senses when making school environmental proposals to achieve a dialogue between what is known and what is learned, in particular contexts.

PALABRAS CLAVE: Educación rural y urbana, enseñanza de las ciencias (nivel primario), educación ambiental, intercambio cultural, ambiente, naturaleza, problemas ambientales, las representaciones sociales y los micromundos.

KEYWORDS: Rural and urban education, science education (elementary level), environmental education, cultural exchange, environment, nature, environmental issues, social representations and microworlds.

1 avelasco@pedagogica.edu.co; arcevel@gmail.com. 
MEMORIAS DEL I CONGRESO NACIONAL DE INVESTIGACIÓN EN

ENSEÑANZA DE LA BIOLOGÍA. VI ENCUENTRO NACIONAL DE INVESTIGACIÓN EN ENSEÑANZA DE LA BIOLOGÍA Y LA EDUCACIÓN

AMBIENTAL. ISSN 2027 1034. P. P. 233-242.

\section{INTRODUCCIÓN}

A partir de lo que se investiga se promueve acciones y proyectos de educación ambiental, por ello cobra importancia la identificación de los sentidos, significados y espacios relevantes de ambiente y naturaleza (micromundos) en estudiantes de zonas rurales y urbanas de los grados cuarto y quinto de primaria.

La descripción de los micromundos relacionados con ambiente y naturaleza, se hace desde lo que resulta relevante en actividades escolares para los estudiantes de cuarto y quinto de primaria en tres escuelas, una rural, una urbana y otra que puede tener ambas connotaciones.

La pregunta que orienta este estudio es: ¿Qué caracteriza y diferencia los micromundos relacionados con los conceptos de ambiente y naturaleza en estudiantes de grados $4^{\circ}$ y $5^{\circ}$ de instituciones Rurales y Urbanas?

La metodología se orienta desde la investigación cualitativa con herramientas de la Investigación - acción. El trabajo se desarrolla en cinco fases: la revisión de referentes conceptuales, el diagnóstico situacional, el diseño y la aplicación de actividades, el análisis y sistematización y la propuesta guía de trabajo en el aula.

Para el análisis de la información se constituyen categorías próximas desde las emergencias relevantes de las actividades. En cada institución surgen ciertos énfasis particulares, que se desprenden de las características propias, del territorio donde prima el mundo de lo urbano o el mundo de lo rural; los micromundos categorizados se describen así:

- Relativos al ambiente: concepciones, elementos que lo conforman, las problemáticas ambientales (entre ellas la destrucción del ambiente, las enfermedades, las relaciones interpersonales, los problemas ocasionados por fenómenos naturales), el territorio y las creencias que provienen de otras regiones.

- Relativos a la naturaleza: concepciones, elementos que la caracterizan, los fenómenos naturales y el mundo de lo vivo.

El estudio teórico del proyecto, se orientó desde el planteamiento de los micromundos, las representaciones sociales, los conceptos de ambiente, naturaleza y territorio.

\section{Micromundos}

El concepto de micromundo fue pensado Seymour Papert; para que los niños pudieran generar y evolucionar estructuras cognitivas, en la perspectiva de Piaget.. Son útiles para aprender acerca de los mecanismos que determinan la 
BIO -GRAFÍA ESCRITOS SOBRE LA BIOLOGÍA Y SU ENSEÑANZA. EDICIÓN EXTRA-ORDINARIA.

MEMORIAS DEL I CONGRESO NACIONAL DE INVESTIGACIÓN EN

ENSEÑANZA DE LA BIOLOGÍA. VI ENCUENTRO NACIONAL DE INVESTIGACIÓN EN ENSEÑANZA DE LA BIOLOGÍA Y LA EDUCACIÓN AMBIENTAL. ISSN 2027 1034. P. P. 233-242.

forma de pensar, las estrategias cognitivas y las representaciones mentales de la realidad. (PALACIOS, 2008).

En la propuesta de (MATURANA, 2002), plantea la formación de los sistemas sociales (es el producto de las interacciones que se dan entre los seres vivos que conforman una sociedad) desde lo biológico y se caracteriza en tres sentidos: Red de Interacción (permite que se establezca un sistema social con unas características particulares), Autoconciencia (como el centro de la formación de ese sistema humano) y Reflexión en el lenguaje (nos lleva a ver el mundo en que vivimos y a aceptarlo o rechazarlo conscientemente).

Un micromundo, desde la perspectiva de (VARELA, 1996) es "Un espacio donde un ser humano se constituye como tal en un sistema social, donde hay una recurrencia en una interacción de cooperación y se establece una autoconciencia"; en un acoplamiento que se da espontáneamente.

En relación con lo mencionado Varela, 1998 citado por (OJEDA, 2001) afirma: "El mundo no es algo que nos haya sido entregado, es algo que emerge a partir de cómo nos movemos, tocamos, respiramos y comemos. Esto es lo que denomino la cognición como enacción, ya que la acción connota el producir por medio de una manipulación concreta".

Es decir que en la medida en que interactuamos en un grupo y espacio determinado, es que constituimos el mundo, en este caso el mundo de los estudiantes emerge en las recurrencias que se presentan en las interacciones en diferentes grupos, pero además en el reconocimiento de lo que tiene significado y sentido para ellos.

Los micromundos son colectivos, es decir, se comparten y construyen con otros, la constitución de identidades tiene que ver precisamente con la posibilidad de encontrarse con el otro y de internalizar su ambiente a través de la experiencia. Así, los micromundos como pautas de acción, se configuran como espacios de interacción, pero también como espacio semántico simbólico, según Maturana (2002).

Además de los micromundos existen otras posiciones con diferente tipo de globalidad y de sentido más relacionadas con el manejo de conceptos y otros más amplios como las representaciones que se plantean respecto a la enseñanza y aprendizaje de los mismos. . Por la amplitud del concepto de micromundos en cuanto a significado y sentido, y por la forma de aprendizaje desde la en acción lo preferimos ante los otros.

El tener en cuenta lo que el alumno sabe a la hora de enseñar, se le nomina y define de múltiples maneras: (VELAZCO, 1991), Flores (2009) citado en 


\section{MEMORIAS DEL I CONGRESO NACIONAL DE INVESTIGACIÓN EN \\ ENSEÑANZA DE LA BIOLOGÍA. VI ENCUENTRO NACIONAL DE INVESTIGACIÓN EN ENSEÑANZA DE LA BIOLOGÍA Y LA EDUCACIÓN AMBIENTAL. ISSN 2027 1034. P. P. 233-242.}

(LACOLLA, 2005), Pozo (2005), Driver \& Esley (2009), proponen la expresión "Concepciones alternativas", considerándola la más adecuada porque involucra una visión "ideográfica", en cambio (CUBERO, 1989) prefiere el término "ideas". Según lo expresa Moscovici en (1973, citado por (DUVEEN, 2003) y (1973 Citado en (LACOLLA, 2005) "La teoría de las representaciones sociales trata de explicar la diferencia entre el ideal de un pensamiento conforme a la ciencia, la razón y la realidad del pensamiento del mundo social".

Cuando se habla de ambiente, surgen varios términos desde las ciencias naturales y humanas, autores como H. Perloff (1973), O. Dollfus (1975), P. George (1979) y R. Méndez (1988) emplean con el mismo sentido diversos términos (COZZANI, 1991).

Existen varios enfoques alrededor del ambiente el enfoque geográfico según (COZZANI, 1991), El ambiente como representación social Azevedo 1999 citado en (SILVA, 2002), (REIGOTA, 1995.) clasificó las representaciones sociales del ambiente en tres grupos: naturalista cuando evidencia elementos naturales, englobando aspectos físicos y químicos, el aire, los otros seres vivos (fauna y flora); globalizante muestra las interacciones entre los aspectos sociales y naturales; y antropocéntrica cuando presenta la utilidad de los recursos naturales para la sobrevivencia de los seres humanos.

Desde un enfoque sistémico, el ambiente no es la ecología, "sino el campo de relaciones entre la naturaleza y la cultura, de lo material y lo simbólico, de la complejidad del ser y del pensamiento; es un saber sobre las estrategias de apropiación del mundo y la naturaleza a través de las relaciones de poder que se han inscrito en las formas dominantes de conocimiento" (LEFF ZIMMERMAN, 2004.)

La palabra ambiente, explica las "circunstancias" o accidentes que rodean la vida, hace alusión a otras condiciones externas que la afectan, considerándola como un transcurrir y reconociendo que hay otros factores, además del medio, que hacen posible o no su desarrollo. Este término, que encierra una noción de dinamismo, de evolución, en tanto las circunstancias son variables a través del tiempo y diferentes según el lugar que ocupan los hombres en el espacio, en la estructura social, etc., implica que los factores o elementos involucrados en el vivir no solamente pueden influir en los grupos humanos, sino que ellos mismos son modificados, humanizados en el contacto (COZZANI, 1991).

El concepto de territorio, se ha definido desde diferentes perspectivas, enfoques teóricos y metodológicos; cuando hablamos de territorio, nos referimos al poder que ejercen los diferentes actores sociales sobre el espacio, delimitándolo y diferenciándolo. 
MEMORIAS DEL I CONGRESO NACIONAL DE INVESTIGACIÓN EN

ENSEÑANZA DE LA BIOLOGÍA. VI ENCUENTRO NACIONAL DE INVESTIGACIÓN EN ENSEÑANZA DE LA BIOLOGÍA Y LA EDUCACIÓN

AMBIENTAL. ISSN 2027 1034. P. P. 233-242.

El territorio es el resultado de la apropiación y valoración de un espacio determinado (García, 1976). El territorio implica prácticas de control y dominio sobre el espacio y una condición de circunscripción territorial que la expresa 0 simboliza; al mismo tiempo el territorio es definido en función de lo que colectivamente se consideran las vivencias, nociones y valoraciones compartidas y a él ligadas; desde esta perspectiva el territorio implica una delimitación simbólica que establece los adentros y los afueras en los sentidos de identificación de las gentes. Por ello, el territorio es pensable y comprensible en la medida en que se conozcan y descifren los contenidos y las maneras como los sujetos viven, experimentan, imaginan, piensan proyectan o inscriben sus sentimientos de pertenencia, intereses, prácticas y poderes en él. (GARCÍA, 2006).

Las perspectivas acerca del significado de Naturaleza son muy diversas, éstas cambian de acuerdo a las condiciones históricas, socioculturales y a las características del contexto, a su vez cada persona maneja varias concepciones, su adopción y aplicación está determinada por las situaciones que se presenten en la cotidianidad.

Según lo propuesto por Eduardo (GUDYNAS, 1999) la naturaleza ha sido invocada como el origen de la riqueza de un país, pero también como un medio salvaje y peligroso, donde lluvias, terremotos u otros desastres deben ser controlados; se ha relacionado como sinónimo de otros conceptos entre ellos madre tierra (al proveer de alimentos), reino salvaje (al hablar de los primeros exploradores), ecosistema o ambiente.

Así pues, es posible hablar de múltiples concepciones las cuales han sido identificadas por diversos autores en estudios que se centran en entender las ideas relacionadas con el concepto naturaleza, como: Concepción Antropocéntrica, como biodiversidad o como el conjunto de las cosas naturales, Concepción Utilitarista, Naturaleza Mercantilista o naturaleza como capital, Perspectiva teológica, Concepción estética o romántica, Naturaleza como sinónimo de ambiente, Naturaleza silvestre, Concepción antropomórfica, La naturaleza como sistema y La naturaleza como organismo.

\section{La Metodología}

La investigación se desarrollo con estudiantes de zonas rurales y urbanas de los grados cuarto y quinto de primaria de tres colegios: La escuela San Francisco en Choachi ( 3 estudiantes de grado $4^{\circ}, 2$ estudiantes de grado $5^{\circ}$ ), el Instituto Educativo Distrital el Verjón Bajo (29 estudiantes de grado $4^{\circ}, 28$ estudiantes de grado $5^{\circ}$ ), y el Instituto Educativo Distrital Campestre Monte Verde de Bogotá (24 estudiantes de grado $4^{\circ}, 30$ estudiantes de grado $5^{\circ}$ ). 
MEMORIAS DEL I CONGRESO NACIONAL DE INVESTIGACIÓN EN

ENSEÑANZA DE LA BIOLOGÍA. VI ENCUENTRO NACIONAL DE INVESTIGACIÓN EN ENSEÑANZA DE LA BIOLOGÍA Y LA EDUCACIÓN

AMBIENTAL. ISSN 2027 1034. P. P. 233-242.

La perspectiva de Investigación acción se orientó desde el planteamiento de (SAGASTIZABAL, 2002) quien la define como "un proceso que genera una serie de competencias entre las que se encuentran: la capacidad de comprender la organización como un sistema, reconocer y jerarquizar los problemas, superar las visiones simples y esteriotipadas de la realidad, evaluar las soluciones, construir conocimientos y equipos cooperativos de trabajo y generar autonomía en la toma de decisiones por parte de la comunidad."

La metodología se desarrolló en cinco fases, que se describen a continuación:

Fase 1. Revisión de referentes conceptuales: Realizar un estudio teórico comparativo acerca de los conceptos relacionados con las representaciones y micromundos de los estudiantes, para establecer los elementos fundamentales para el análisis de la información.

Fase 2.Diagnóstico situacional: Caracterizar la población objeto de estudio, así como los sentidos y significados que circulan en relación a las problemáticas ambientales relevantes para ellos en el contexto rural y urbano.

Fase 3. Diseño y aplicación de actividades: diseñar y aplicar actividades de trabajo en el aula de clase que permitan conocer el contexto de los escolares y reconocer los micromundos relacionados con los conceptos ambiente y naturaleza.

Fase 4. Análisis y sistematización: categorizar y analizar la información obtenida en la fase de campo.

Fase 5.Propuesta guía de trabajo en el aula: proponer una estrategia pedagógica de trabajo en el aula, que vincule el entorno inmediato de las instituciones y las problemáticas ambientales relevantes para los estudiantes en los contextos rurales y urbanos.

La información se recoge a través de actividades de clase donde se exponen situaciones relacionadas con dichos conceptos, como: fotos de lugares para reconocer el entorno próximo, clasificación de imágenes de lo no natural y lo natural, planteamiento de problemas que exigen la toma de decisiones en situaciones de conflicto que involucraban su territorio, en juegos donde se narraban anécdotas y en la elaboración de un mapa del recorrido de la casa a la escuela.

\section{RESULTADOS}

Encontrados los micromundos relacionados con el ambiente existen varias formas de nombrarlo: se describe desde los elementos que lo componen, se evidencia en las dinámicas internas, (los cambios en dichos elementos, el reconocer el ambiente en que viven los organismos, de donde provienen elementos como el agua y las relaciones que establecen con el alimento) en 


\author{
MEMORIAS DEL I CONGRESO NACIONAL DE INVESTIGACIÓN EN \\ ENSEÑANZA DE LA BIOLOGÍA. VI ENCUENTRO NACIONAL DE \\ INVESTIGACIÓN EN ENSEÑANZA DE LA BIOLOGÍA Y LA EDUCACIÓN \\ AMBIENTAL. ISSN 2027 1034. P. P. 233-242.
}

las cuales se involucran los elementos del territorio. "El medio ambiente para mí son los árboles, la naturaleza, las flores y así lo que hay en el campo". "El viento, el agua, el sol, los planetas, el sistema solar"; "a veces hay sol, a veces nublado, hay una quebrada que a veces se seca por el sol".

Algunos grupos muestran que para ellos naturaleza y ambiente pueden ser equivalentes. "el ambiente son las plantas, los animales, el agua y la naturaleza", "el ambiente es como toda la naturaleza".

Las problemáticas ambientales que reconocen están muy relacionadas con sus vivencias en el territorio, por ejemplo en el Verjón una problemática sentida es el acceso y uso del agua, en Monteverde el uso del agua y la inseguridad (representada por pandillas, atracos, violaciones, peleas, expendio de drogas). "Cuando no hay agua cierran el colegio", "si uno bota el agua, al otro día traemos una bolsa de agua". "Acá en el barrio hay hartas pandillas..." "Aquí... apenas se encuentra en las cancha se dan..."

Las relaciones sociales se configuran en tres escenarios: la familia, la escuela y el barrio, en el caso de Monteverde, en el Verjón y San Francisco se reconocen otros espacios como diferentes al barrio por ser zonas que están más alejadas de la ciudad, como el parque de las moras.

Las familias difieren en cuanto a su estructura pues en las dos últimas las familias tienen más tendencia a ser nucleares.

En cuanto a las relaciones en la familia, los niños de la ciudad permanecen mucho menos tiempo con sus padres, escuchan peleas frecuentes en su casa por motivos económicos. En el caso de las escuelas rurales los padres permanecen más cerca y tienen reglas muy claras que los niños obedecen, las discusiones se dan alrededor de los oficios que deben cumplir. "yo ayudo a organizar a barrer lavo los platos tiendo la cama de mi mamá y todos los días tiendo la mía, organizo mi cuarto y ayudo".

En la escuela se muestra que las relaciones con los docentes son de cordialidad y respeto especialmente en las escuelas del Verjón y San Francisco, en Monteverde hay quejas de los maestros pues algunos estudiantes los irrespetan y ellos les deben llamar la atención.

En todos los casos el territorio es un sitio de exploración y reconocimiento para los estudiantes pues reconocen lugares, en ellos animales, las plantas de la zona son reconocidas sólo por ser alimenticias como las moras silvestres o por ser plantas de cultivo, sitios para recrearse, sitios peligrosos. "por allí abajo le decimos a un potrero donde hay muchas moras el parque de moras". Además de lo anterior reconocen las dinámicas de cambio que se han venido operando (nuevas construcciones, calles, tala de árboles). 


\author{
MEMORIAS DEL I CONGRESO NACIONAL DE INVESTIGACIÓN EN \\ ENSEÑANZA DE LA BIOLOGÍA. VI ENCUENTRO NACIONAL DE \\ INVESTIGACIÓN EN ENSEÑANZA DE LA BIOLOGÍA Y LA EDUCACIÓN \\ AMBIENTAL. ISSN 2027 1034. P. P. 233-242.
}

Es de resaltar que son los niños quiénes muestran más conocimiento de los espacios, de los lugares donde pueden desarrollar sus juegos, sus aventuras, donde se encuentran animales, cursos de agua, pozos entre otros.

Los fenómenos naturales se explican desde un desequilibrio de la tierra, acentuado por las acciones antrópicas sobre el ambiente (como la contaminación, la tala de bosques y las basuras). "Antes en las calles había naturaleza y ahora hay cemento y todo eso", "Digamos todo esto era bosque, y lo tumbaron y hicieron una vía",

Otra respuesta frecuente, vincula los fenómenos naturales con lo religioso como castigos a los malos comportamientos humanos. "Dios manda los terremotos porque los hombres matan"

La naturaleza se concibe para algunos como una creación de Dios, también desde algunos de los elementos, donde se reconoce que no es posible que se puedan elaborar por parte de los seres humanos. En otros casos los imaginarios acerca de ciertos lugares que están ligados a creencias significan y regulan las actuaciones en el territorio, así como creencias traídas por sus padres.

La naturaleza se manifiesta al igual que el ambiente en una dinámica permanente tanto en lo referente a lo vivo como a los componentes no vivos.

Lo no natural se define desde la negación de lo natural, por ejemplo si se dice que lo natural está relacionado con lo vivo, lo no natural será lo que no está vivo. "Lo que no tiene vida... Los carros, una ciudad, un equipo". "Los carros, el piso, las maquinas... Porque no viven." Sin embargo se encuentran contradicciones cuando el objeto proviene de un organismo.

Una evidencia importante es el reconocimiento de la naturaleza desde los olores de animales y plantas, lo que muestra un reconocimiento sensitivo de la misma, que se asocia con una relación placentera.

Los objetos escogidos son naturales porque tienen vida, porque nacen, crecen, se reproducen y mueren, porque respiran, por ser etapas del ciclo de vida.

\title{
CONCLUSIONES
}

Los micromundos se expresan de múltiples formas, son influenciados por el territorio y por las experiencias.

Es de resaltar que las categorías se constituyen como una forma de organizar la información, pero los micromundos de los niños se constituyen en la medida en que se operan dinámicas de relaciones entre las diferentes formas de actuar y pensar, las cuales pueden ser contradictorias entre sí, pero que sólo se evidencian en la medida en que se realizan procesos para visibilizarlas y relacionarlas. Ello muestra que los micromundos no son estáticos, ni 
MEMORIAS DEL I CONGRESO NACIONAL DE INVESTIGACIÓN EN

ENSEÑANZA DE LA BIOLOGÍA. VI ENCUENTRO NACIONAL DE INVESTIGACIÓN EN ENSEÑANZA DE LA BIOLOGÍA Y LA EDUCACIÓN

AMBIENTAL. ISSN 2027 1034. P. P. 233-242.

perspectivas fijas e inmodificables y que su construcción se alimenta desde múltiples experiencias de los individuos.

Es necesario conocer el contexto de las Instituciones puesto que ello influye en las concepciones de los individuos acerca de ambiente y naturaleza. Aspectos como: La clase de ciencias, la actividad económica que desempeñan sus padres, las actividades extracurriculares y el territorio en el cual se desenvuelven, entre otras.

La perspectiva de los micromundos orienta la construcción de una propuesta educativa donde se hace relevante construir conocimiento a partir de las diferentes experiencias que constituyen el mundo de los estudiantes, "El mundo no es algo que nos haya sido entregado: es algo que emerge a partir de cómo nos movemos, tocamos, respiramos y comemos" (VARELA, 1996, P. 6), es decir que el profesor debe movilizar los saberes de los estudiantes, desde su propio contexto, teniendo en cuenta sus perspectivas acerca de naturaleza, ambiente y territorio.

\section{BIBLIOGRAFÍA}

- Bermúdez, O. (2003). Cultura y ambiente: la educación ambiental, contexto y perspectivas. Bogotá, Colombia: Universidad Nacional de Colombia. Instituto de Estudios Ambientales.

- Cozzani, M. R. (1991). El concepto de medio ambiente humano en geografía. Revista de Geografía Norte Grande , 18, 75-78.

- Cubero, R. (1989). Cómo trabajar con las ideas de los alumnos. Sevilla:: Diada.

- Duveen, G. y. (2003). epresentaciones sociales. Problemas teóricos y conocimientos infantiles: La presentación de las representaciones sociales: dialogo con Serge Moscovici". Barcelona: Gedisa.

- Flores, F. e. (2009). Recuperado el 22 de Julio de 2009, de http://ideasprevias.cinstrum.unam.mx:2048/index.php

- García, C. (2006). Las representaciones sociales del territorio, enfoque y metodología para su estudio. Colombia: Grupo de Investigación en Territorio, Universidad de Antioquia, Instituto de Estudios Regionales.

- Gudynas, E. (1999). Imágenes, ideas y conceptos sobre la naturaleza en América Latina. Recuperado el 14 de Octubre de 2011, de http://www.gudynas.com/publicaciones/capitulos/GudynasConceptosNat uralezaCo10.pdf 
MEMORIAS DEL I CONGRESO NACIONAL DE INVESTIGACIÓN EN

ENSEÑANZA DE LA BIOLOGÍA. VI ENCUENTRO NACIONAL DE INVESTIGACIÓN EN ENSEÑANZA DE LA BIOLOGÍA Y LA EDUCACIÓN

AMBIENTAL. ISSN 2027 1034. P. P. 233-242.

- Lacolla, L. (JULIO de 2005). Representaciones sociales: Una manera de entender las ideas de nuestros alumnos. Recuperado el 24 de Julio de 2010

- Leff Zimmerman, E. (Diciembre de 2004.). Educación Ambiental: Perspectivas desde el Conocimiento, la Ciencia, la Ética, la Cultura, la Sociedad y la Sustentabilidad. Revista Ideas ambientales: .

- Maturana, H. (2002). Transformación en la convivencia. Santiago, Chile: Dolmen.

- Ojeda, C. (2001). Francisco Varela y las ciencias cognitivas. Rev.Chilena de Neuro-psiquiatría. , 39 (4), 286-295.

- Palacios, J. (6 de Noviembre de 2008). Juan Palacios Management con conciencia interdependiente. Recuperado el 14 de Octubre de 2011, de Micromundos tricerados: http://jpalaciosgil.blogspot.com/2008/11/micromundos-tricerados.html

- Reigota, M. (1995.). Meio ambiente e representaçao social.

- Sagastizabal, M. y. (2002). La Investigación Acción como estrategia de cambio en las organizaciones. Buenos Aires: La Crujía Ediciones.

- Silva, R. L. (2002). Representaciones sociales de medio ambiente y educación ambiental en docentes universitarios. Tópicos en Educación Ambiental , 4 (10), 22-36.

- Varela, F. (1996). Ética y Acción. Santiago,, Chile: Dolmen.

- Velazco, j. M. (1991). ¿Cuándo un ser vivo puede ser considerado animal? Análisis de las concepciones alternativas del alumnado acerca del significado de "animal". Enseñanza de las ciencias. , 9 (1), 43. 